ARTIGOS

\title{
IGUALDAD DE GÉNERO Y ENFOQUE POR COMPETENCIAS: DIFICULTADES EN LA FORMACIÓN DEL PROFESORADO
}

Ramón PACHECO

Universidad de Sevilla -US

Sevilla - España

ramongp@us.es

https://orcid.org/0000-0001-8901-7068

Elena ZUBIAURRE Universidad Alfonso X El Sabio

Madrid - España

ezubiiba@uax.es

https://orcid.org/0000-0002-1474-9464

\begin{abstract}
Resumen: La generalización del enfoque competencial desde la formación obligatoria impartida al futuro profesorado del sistema educativo español aparece fundamentada técnicamente, acompañada de una pretendida vocación de neutralidad que lo convierte en incontestable, pero que solapa una lógica procedimental muy dependiente de la puesta en práctica; ésta, creemos, favorece la merma de la capacidad reflexiva de los sujetos del conocimiento. Ello contribuye al afianzamiento de representacionesdiscriminantes con las mujeres, pasando inadvertidas para las herramientas metodológicas que el mismo enfoque ofrece a la comunidad educativa. Aquí defendemos que este enfoque toma parte fundamental de un proyecto mayor promovido por organismos internacionales, para el despliegue de un modelo ideológico neoliberal por vía de la institucionalizaciónde una racionalidad cognoscitiva. Igualmente, denunciamos la impotencia del modelo frente a los mandatos de género, por su incapacidad para acceder a los planos en los que los significados atribuidos sociohistóricamente a mujeres y hombres devienen canónicos.
\end{abstract}

Palabras-clave: Competencias.profesorado.formación.género.representaciones

\section{GENDER EQUALITY AND COMPETENCY-BASED APPROACH: ISSUES OF TEACHER EDUCATION}

\begin{abstract}
Competency-based approach is generalized through compulsory training given to future teachers in Spain. This approach is technically based, as a guarantee of supposed ideological neutrality that makes this perspective unquestionable. However, a procedural logic dependent on implementation is overlapped by the competences, which manages to limit the reflective capacity of the subjects in the information society. This initiative contributes to the strengthening of gender representations that discriminate against women and tend to go unnoticed by the methodological tools that are offered. We defend that competency-
\end{abstract}




\section{autêntica}

DOI https://doi.org/10.31639/rbpfp.v12i24.356

based approach takes a fundamental part of a larger project promoted by international organizations, for the deployment of a neoliberal ideological model through the institutionalization of a cognitive rationality. We also denounce the model inability to question gender mandates, due to its dependence on implementation and consequent impossibility to explore the levels in which the meanings sociohistorically attributed to women and men become immutable.

Keywords: Competences. teachers training. gender. representations

\section{IGUALDADE DE GÊNERO E ABORDAGEM DE COMPETÊNCIAS: ANÁLISE DE DIFICULDADES PARA A FORMAÇÃO DE PROFESSORES}

Resumo: A generalização da abordagem de habilidades, uma vez que a formação obrigatória de futuros professores da educação nacional espanhola é tecnicamente baseada; parece estar acompanhada de uma alegada vocação de neutralidade, tornando-a indiscutível, ao mesmo tempo em que oculta uma lógica processual muito dependente da implementação. Acreditamos que isso promove a redução da capacidade reflexiva dos sujeitos do conhecimento. Tudo isso contribui para a consolidação de representações discriminatórias contra as mulheres, que passam despercebidas pelos instrumentos metodológicos favorecidos por esse discurso. Aqui argumentamos que essa abordagem é parte fundamental de um projeto maior promovido por organizações internacionais para a implantação de um modelo ideológico por meio da institucionalização da racionalidade cognitiva. Além disso, denunciamos a impotência desse modelo diante dos mandatos de gênero, por sua incapacidade de acessar níveis em que os significados atribuídos sócio-historicamente a mulheres e homens se tornam canônicos.

Palavras-chave: Competências, Formação de Professores, Gênero, Representações 


\section{autêntica}

DOI https://doi.org/10.31639/rbpfp.v12i24.356

\section{Introducción. El enfoque por competencias en el proyecto liberal de reuniformización del conocimiento}

La incorporación de la retórica competencial en los discursos pedagógicos entre quienes aspiran a iniciar la carrera docente en España, a través del curso del Máster en Formación del Profesorado de Educación Secundaria Obligatoria y Bachillerato, Formación Profesional y Enseñanza de Idiomas, es una tónica general. No queremos decir con ello que todo docente en ciernes emplee del mismo modo el término "competencia", pero sí pretendemos subrayar su recurrencia en las argumentaciones, instrumentos metodológicos (sobre todo aquéllos con pretensión cuantificadora) y conclusiones de que se sirve el alumnado. También señalaremos, desde nuestra mera experiencia, que dicha recurrencia en el empleo del término "competencia" parece indistinta a estudiantes provenientes de titulaciones encuadrables en cualquier disciplina académica, así como al género, lo que parece arrogarle, a priori, cierta propiedad uniformadora.

A lo largo del siglo XX y las dos primeras décadas del XXIse ha desarrollado tal diversidad de disciplinas que se ha convertido en habitual que algunas compartan -o parezca que lo hacen- objeto de estudio. En este sentido, más clarificadoras son, respecto a las clásicas retrospectivas, las muy diversas orientaciones prospectivas que distintas disciplinas pueden asumir ante un mismo problema u objeto de estudio. Tamaña diversidad de disciplinas invita a pensar que éstas plantean formas igualmente heterogéneas de entrar en contacto con el conocimiento; ante ello, el discurso de las competencias se presenta como agente uniformador, en su propuesta de estándares que parecen ignorar por completo las viejas tensiones entre nominalismo y realismo, razón y emoción, descripción, explicación y comprensión, razón intemporal y razón histórica, estas últimas bastante explicativas de cómo el conocimiento se crea, se destruye, se supera, se complementa, se debate, se critica, surgiendo de esas dinámicas planteamientos paralelos, contradicciones, paradojas, tensiones. Por ello, a pesar de la retórica constructivista con que se presentan envueltas las competencias (fundamentalmente, a partir del proyecto DeSeCo, en 1997), consideramos ciertamente improbable que el profesorado en formación inicial logre apropiarse de dinámicas educativas planteadas en clave competencial en torno a los ejes de la construcción y la interpretación del conocimiento, pues, dependientes de cumplir con los requerimientos técnicos de una puesta en práctica, éstos se verán inevitablemente sustituidos por los de la producción y lectura (decodificación) del mismo.

Aunque la epistemología de la llustración nos abre las puertas de un nuevo régimen societal gobernado por la ciencia, es sabido que ésta sufre diferentes procesos de diversificación y emancipación históricamente coherentes, entre los que resultan especialmente significativos, por sus trayectorias en ocasiones errantes y poco dadas a la progresión, aquéllos vividos por las emergentes ciencias sociales: modernismo positivista, nominalismo, estructuralismo-funcionalismo, cognitivismo, psicoanalismo, postmodernismo, constructivismo, interaccionismo, culturalismo. Ello evidencia que no sólo las distintas formas de clasificar las ciencias implican diversos modos de entablar contacto con el conocimiento, sino que una misma disciplina puede encerrar una amplia variedad de alternativas epistemológicas y metodológicas para una diferencial validación de aquél. Célebre es, en esta línea, la cita en la que Vygotsky defendía la existencia de varias psicologías. Una perspectiva sociohistórica de la ciencia permite entender sin demasiado esfuerzo que la construcción del conocimiento no ha seguido hasta nuestro días sendas unilineales por cada disciplina, pautadas éstas por la mera acumulación de contenido, sino que el conocimiento se ha ido conformando, nunca de forma definitiva, entre debates, tensiones y controversias, en el curso de los cuales, muy particularmente en el caso de las ciencias sociales, los problemas que se iban planteando giraban en torno a cuestiones primarias como la mera delimitación de los sujetos y de los objetos. Por ello hablamos de conocimiento y no de contenido escolar, habida cuenta que la vocación del proyecto de las competencias no es la de seleccionar el contenido, radicando ahí además su pretendida neutralidad ideológica, sino la de "hacer pensar" en clave competencial. 


\section{autêntica}

DOI https://doi.org/10.31639/rbpfp.v12i24.356

No deja de resultar curioso que esas dinámicas precipitasen el surgimiento de un feminismo con capacidad para "percibir mejor la no racionalidad de nuestra sociedad racional", inspirador de la "reflexión sobre la relación entre el patriarcado y la producción en una sociedad ya gobernada por la lógica de las ciencias y las tecnologías", que nos llevaría a cuestionar la neutralidad científica no ya de los sujetos sino del contenido científico mismo, por ejemplo "hasta qué punto la lógica de las matemáticas y de las ciencias podría ser producto de una cultura patriarcal" (Fourez, 1994). De este modo, el feminismo (también el ecologismo) evidencia lo que ya describieron Giddens (1991) o más tarde Castells (1997), esto es que el conocimiento no es el único problema de su homónima y emergente sociedad, que lo son también los nuevos sujetos del mismo, empoderados por las nuevas tecnologías, actuando desde una reflexividad.

En este difuso marco global que dibujan los flujos de información, abandonando ya la referencia espacio-territorial de los estados-nación (Castells, 1997), tiene cabida mostrar cautela frente a las propuestas políticas orientadas a la uniformización universal del conocimiento, como sucede con aquéllas emanadas del enfoque por competencias. Estas propuestas, promovidas en este caso por organismos supraestatales, deberán partir necesariamente de estándares que, a diferencia de lo que sucedía en las sociedades analógicas, no pueden ser ya socialmente implementados desde su misma puesta a disposición por vía legislativa por parte de los productores oficiales del conocimiento, sino que tendrán que afrontar en primer lugar las muy heterogéneas formas de apropiación de los nuevos sujetos, lo que plantea, al menos, dos contradicciones: por un lado, si bien el paradigma científico-tecnológico sigue funcionando como garante de los agentes de producción del conocimiento, el acceso generalizado a la información y su organización en red favorecen que un mismo contenido, simultáneamente, ocupe diferentes espacios, realice todo tipo de trayectorias, impregne múltiples discursos, se presente como mediador de actividades prácticas llevadas a cabo cooperativamente, sufriendo transformaciones constantes en cada uno de estos procesos que son del todo comprometedoras de su presentación como estándar; por otro, el proceso mismo de apropiación entraña reflexividad, en otras palabras el conocimiento, en una sociedad como la actual, no puede ser asimilado por la vía directa sino que debe ser problematizado, comparado con otras formas accesibles de conocimiento y, de este modo, sometido constantemente a validaciones críticas que comprometerán la "sacralidad" de que le dotan las fuentes legítimas que lo producen, incluyendo las vías estables de afirmación representadas por el consumo.

\section{Competencias, conocimiento práctico y reflexividad}

Conviene comenzar este punto realizando algunas matizaciones preliminares. En primer lugar, no descuidamos el hecho de que la producción oficial del conocimiento siga acaparada por los hombres: no sólo siguensiendo muchos más en número, sino que, sobre todo, la conciliación representacional masculina de los modelos científico-tecnológicos sigue gozando de excelente salud. En un contexto de despliegue total del liberalismo económico, ello tendrá efectos particularmente perversos sobre las mujeres, quienes, fuera del movimiento feminista, se sentirán "menos" sujetos, al tiempo que afrontarán todo tipo de dificultades para justificar esta realidad sin que el peso de la culpa acabe por inmovilizarlas. Por otro lado, hablamos de formas de apropiación del conocimiento que son privilegiadas, las cuales incluyen no sólo la dimensión procedimental necesaria para su conceptualización racional como técnica, sino otra actitudinal que interviene en el trascendental plano de las emociones, para las que históricamente se ha socializado diferencialmente a hombres (actitudes para desenvolverse en el espacio público) y mujeres (actitudes propias del espacio doméstico).

Nuestra tesis defiende que, con objeto de doblegar a estas contradicciones, las propuestas uniformizadoras son formuladas procedimentalmente, es decir definen conjuntamente a conocimiento y puesta en práctica, lo que permite formalizar a esta última como técnica oficial y legítima para llegar a aquél, que queda 


\section{autêntica}

DOI https://doi.org/10.31639/rbpfp.v12i24.356

reconceptualizado como saber específico. De este modo, este último queda determinado ahora por un ámbito de aplicación que es contextual, es decir, por un lado estándar y por otro estático, impermeable a las definiciones situacionales alternativas planteables por los sujetos.

Son numerosos los trabajos que ponen de manifiesto las ventajas de los saberes, conceptualizados del modo en que acabamos de ver, para alimentar una lógica productiva laboral basada en la mera transmisión de éstos (DELAY, 2006), habiendo incluso quienes defienden enérgicamente su implantación en la escuela (OUELLET, 2007). El enfoque por competencias representa probablemente la mejor muestra del calado de estas iniciativas. Tal proyecto únicamente puede articularse en torno a la creación de toda una arquitectura cognoscitiva, cuya implementación debe correr a cargo de una institución educativa muy extendida, por un lado, en el espacio público, diluyendo los muros de los centros educativos para hacer a éstos permeables a las lógicas de producción que regulan el mundo del trabajo; y, por otro, en el tiempo del ciclo vital de las personas, encontrando, como subraya Beck (1998), un nuevo hito transicional en la "educación mínima" que vendrá a sustituir al de la educación obligatoria. Dicha arquitectura cognoscitiva se tornará útil para canalizar, de un modo deseable para los agentes impulsores de estas iniciativas, la propiedad reflexiva de los sujetos. Por el momento, nos limitaremos a afirmar que ningún proyecto de copado cognoscitivo del espacio público y de los ciclos vitales en modo alguno permite augurar buenos presagios a las mujeres, quienes, paradojas de sociedad patriarcal, son amplia mayoría entre quienes se postulan para integrar el cuerpo funcionarial del profesorado de secundaria y bachillerato. Y, sin embargo, la puesta en práctica de esta arquitectura cognoscitiva requerirá de un profesorado conductor de las nuevas lógicas, al que ya se le presuponen las destrezas para la validación científica del conocimiento, no así la adquisición de las destrezas procedimentales, como acabamos de ver, ahora necesarias para la implantación del modelo. Ello requerirá, cuando menos, su socialización efectiva en el discurso de las competencias, por lo que no es de extrañar que éstas se hayan convertido en piedra angular de la formación inicial.

En realidad, el discurso de la modernidad ha encontrado siempre uno de sus ejes vertebradores en el papel de sujetos y objetos en torno a la construcción del conocimiento (HABERMAS, 1985). Bien es sabido que la empresa de la llustración en ningún momento fue sencilla, pues se trataba de sustituir los principios reguladores de la organización social emanados hasta en ese momento de una autoridad divina, requiriendo por tanto criterios convincentes, efectivos, objetivos, verificables, en resumen inspiradores de un conjunto nuevo de reglas que permitieran una percepción inequívoca, sólida, de una nueva realidad dependiente de un objeto compartido a partir de un significado estable. Con una Revolución Industrial por canalizar en términos de organización social y tras un período inicial de propuestas teóricas para llevar a cabo esta empresa tratando de articular los principios emanados de la razón con los de la divinidad, en los que esta última incluso coexistía asumiendo el rol de científico (El Gran Ingeniero, El Relojero del Cosmos...), las diferentes ciencias sociales, bajo versiones positivistas inspiradas en leyes a imagen y semejanza de las de la naturaleza y en la causalidad newtoniana, comienzan a florecer. Ello da acceso a una sociedad a principios del siglo XX ya por completo articulada, como pone de manifiesto y desarrolla Weber (1998), en torno al trabajo profesional, sobre la que la psicología positivista (conductismo) sólo puede irrumpir triunfante: trabajo profesional y conductismo volverán a aparecer unidos a finales de siglo como ejes fundamentales del nuevo enfoque por competencias, el cual surge en primera instancia para dar continuidad de acuerdo a sus postulados conductistas a la débil e inconsistente pedagogía por objetivos (PÉREZ-GÓMEZ, 2007 y 2008).

Cabe, pues, reconocer haber dejado atrás ese modelo. En la actual sociedad de la información, ni siquiera la comunidad científica puede imponer las formas de validación del conocimiento sin contar con los agentes educativos. Fuera de la realidad institucional escolar, esa equivalencia entre sujetos y objetos es también un 


\section{autêntica}

DOI https://doi.org/10.31639/rbpfp.v12i24.356

hecho. Frente a ello, el proyecto neoliberal europeo ha puesto claramente el foco sobre los sujetos emergentes, seleccionando a la educación como medio principal de actuación: en primer lugar captándolos desde la temporalidad, desarrollando las políticas del lifelong learning y extendiendo al máximo la escolarización obligatoria; en segundo lugar, imponiendo, por la vía oficiosa de las recomendaciones provenientes de organismos internacionales, unos estándares verticales, principalmente las competencias, que operan jerárquicamente a partir de traducciones desde los niveles superiores hasta los inferiores, desde las políticas hasta las aulas, con objeto de canalizar de manera controlada el despliegue de los nuevos sujetos a partir de las formas de apropiación de los mismos y mismas, incluyendo ahí el control sobre las actitudes.

Ello nos mete de lleno en un proyecto de colonización del sujeto promovido por el discurso de las competencias en el que el profesorado es un importante objetivo (MARTíNEZ-BONAFÉ, 2004).Su limpia penetración cognoscitiva, con pretensiones de extenderse hacia el conjunto de la vida social (MARTíNEZ-BONAFÉ, 2004), se puede justificar por el carácter aparentemente técnico y neutral de que le dota su inserción en políticas oficiosas impulsadas por influyentes organismos internacionales como la OCDE (PÉREZ-GÓMEZ, 2008). Más concretamente, "lo que hay detrás y en el fondo del debate sobre la formación del profesorado es una teoría del sujeto y una teoría del conocimiento, y una teoría de la relación entre ambos" (MARTíNEZ-BONAFÉ, 2004). El enfoque por competencias tiene que ver con cómo se gobierna el razonamiento sobre la relación entre sujeto y conocimiento; se trata, por tanto, de intervenir sobre la subjetividad al amparo de un campo institucional (MARTÍNEZ-BONAFÉ, 2004).

La clave de esta colonización del sujeto por parte del discurso de las competencias se encuentra, creemos, en la inmunidad de que se dota al conocimiento frente a la reflexibilidad. Si bien el empleo de la vía cognoscitiva se puede reconocer como un recurso innovador, no hay nada nuevo en que iniciativas de corte liberal encuentren por vocación confundir mercado y medio, con objeto de disolver el estado en aquél. En un plano cognoscitivo que se adentra en la vida cotidiana de las personas, las competencias se introducen como unidad de cambio e intercambio, dando cuerpo a una gramática liberal que presenta además la propiedad de manifestarse representacionalmentey de forma institucionalizada. Es la asunción de esta gramática liberal lo que las blinda frente a la reflexividad de los actores individuales al presentarse autoritariamente como medio, evitando el problema sugerido por Lamo de Espinosa (1990) de que los propios actores que producen las regularidades que dan forma a los sistemas de acciones típicas, esto es institucionalizándolas, las descubran y acaben pautando sus acciones tomándolas en consideración.

Por eso insistimos en el uso del término colonización. No se trata, simplemente, de imponer un discurso unidireccional, sino de llegar al sujeto y colonizarlo con objeto de que éste o ésta se apropie de una manera tipificada y convencionalizada del conocimiento, lo que implica la puesta en marcha de estrategias actitudinales, de ahí su coexistencia junto a la vertiente procedimental en el enfoque por competencias. El o la sujeto, posteriormente, estará en grado de reproducir por sus propios medios formas típicas de adaptación al sistema; esto último tiene lugar desde la privilegiación de formas homogéneas de apropiación, estas últimas ya tecnificadas por ser el resultante en forma de declinaciones de una objetividad legitimada científicamente en la que se incluyen patrones actitudinales igualmente legítimos. De entre estos sujetos, el papel de mayor relevancia corresponde probablemente al profesorado en formación inicial, pues en este caso no sólo encarna el espíritu renovador de las diferentes reformas educativas, sino que de ellos y ellas se espera que ejerzan como traductores privilegiados del discurso de las competencias a alumnado, familias, resto de docentes y equipo directivo del centro. De este modo, las actitudes del profesorado en formación inicial se ven canalizadas, diríamos incluso neutralizadas, por el frenesí implícito al supuesto carácter novedoso de las iniciativas, coaccionando su capacidad de pensarse a sí mismos y mismas en el interior de esas dinámicas. El discurso 


\section{autêntica}

DOI https://doi.org/10.31639/rbpfp.v12i24.356

de las competencias sitúa al conjunto de la cada vez más amplia comunidad educativa ante una ineludible estructura cognoscitiva que depura su capacidad reflexiva para su inserción en un aparato ideológico liberal.

\section{Las mujeres en las configuraciones normativas del espacio social: el discurso competencial profesionalizador frente a la acción ejecutiva del patriarcado}

Otra de las grandes empresas de la modernidad sobre la que cabe poner el foco es la configuración no sólo jurídica sino sobre todo normativa del espacio social, cuestión en la que la distinción hombre-mujer rápidamente se convertirá en articulador principal, no únicamente en la definición de las formas de participación sino también como argumento fundamental para la validación de los sujetos políticos. La vertebración de la sociedad en torno al trabajo profesional servirá para incidir en la separación entre las esferas pública y privada, sentando las bases del patriarcado moderno.Bajo esta distinción subyace, desde un momento muy temprano, una división sexual del trabajo ya moderna, la cual permitirá plantear el espacio de una forma igualmente moderna, esto es social. La división sexual del trabajo permitirá a la teoría social situar al hombre como productor en el espacio público y a la mujer como reproductora en el doméstico o privado (RUBIN, 1975; BOURDIEU, 1998). BUTLER (1990), por un lado, se apoyará en esta distinción para identificar el medio público como aquél en que tienen lugar las políticas corporales sobre las que se asienta la heteronormatividad; por otro, identificará el espacio público con el dominio de las representaciones, las cuales tendrán como base al sujeto.

El carácter social del espacio vendrá conferido, en gran medida, por las simbolizaciones compartidas, que son manifestaciones de los significados (sociales), estos últimos garantes del entendimiento entre los actores que ocupan un escenario para la acción determinado. Aquí nos interesaremos por la producción de estos significados, en especial de aquéllos con vocación normativa y sancionadora. Estos últimos pueden presentarse como prediscursivos (BUTLER, 1990) o, en otro sentido, prelingüísticos (BRUNER, 1991), cuando, emanados desde la autoridad del sistema de organización social con forma de tradiciones, costumbres, valores, leyes (CRAWFORD, CHAFFIN Y FITTON, 1995) o, también, mitos, se articulan para conformar un trasfondo normativo que, por un lado, configura un conjunto de normas canónicas para la acción en ese escenario y, por otro, delimita el escenario mismo, fomentando el establecimiento de ritos de acceso a éstos, por lo general distinguidos por categorías sociales (en especial el género).

Los distintos segmentos espaciales aparecen a partir de aquí emancipables de las delimitaciones realizables bajo los criterios de la ciencia tradicional, particularmente la física, definiéndose los compartimentos sociales que forman por las configuraciones normativas particulares que los gobiernan, estas últimas afirmadas fundamentalmente por medio de lo que autoras como Lutz y Abu-Lughod (1990), en su exitoso esfuerzo por mostrar cómo los discursos desafían o refuerzan las configuraciones normativas proyectadas por las estructuras sociales, denominan políticas emocionales. Así, una misma estancia de hospital puede asumir innumerables configuraciones como compartimento social: si mientras una enfermera aplica cuidados en ella a una paciente entra sin llamar a la puerta un cirujano, la paciente verá confrontadas las normas del espacio doméstico que regulan la actividad que la enfermera, en ese momento, está llevando a cabo, con las del cirujano, este último garante de la ciencia que gobierna el espacio público en una sociedad posindustrial. En este sentido, es de prever que una sonrisa en la enfermera entre dentro de las expectativas de esa paciente, por el importante componente emocional que se atribuye al espacio doméstico, mientras que una interpretación será necesaria si la sonrisa se dibuja en la cara del cirujano, pues el componente racional que se atribuye al espacio público permite tolerar la frialdad (incluso valorarla como profesionalidad). 


\section{autêntica}

DOI https://doi.org/10.31639/rbpfp.v12i24.356

Una idea similar está ya en Barker (1978), quién, aunque mantiene una delimitación física del espacio, logra identificar en ellos reglas de situación que rigen tanto para el discurso como para la acción. El posterior principio de cooperación de Grice (1989), para quién existe un cierto número de máximas que regulan idealmente los intercambios conversacionales, se complementa a la perfección con la propuesta de Barker. La desviación sobre una de esas máximas produce "implicaturas conversacionales", esto es la búsqueda por parte del conjunto de participantes del significado en lo excepcional, "significados que radican en la naturaleza de su desviación respecto al uso corriente" (BRUNER, 1991), por tanto:

Cuando la gente se comporta de acuerdo con el principio situacional de Barker o con las máximas de interacción conversacional de Grice, no preguntamos por qué: sencillamente la conducta se da por supuesta como si no tuviera necesidad de más explicaciones. Como es lo corriente, se experimenta como algo canónico y, por consiguiente, que se explica a sí mismo (Bruner, 1991:65).

Creemos que la aquí defendida naturaleza dual que adoptan los diferentes escenarios sociales hace de éstos la principal vía de afirmación del patriarcado en las sociedades modernas, pues favorece configuraciones normativas sexistas que se repiten e institucionalizan en las puestas en práctica (dimensión ejecutiva) por parte de sujetos mermados de su capacidad reflexiva, y se fundamentan en un trasfondo vertebrado por significados sociales sedimentados por vía sociohistórica, los cuales sirven de soporte para construir representaciones que difieren con frecuencia en función del sexo: mujeres y hombres participan de forma desigual de las tradiciones, reciben atribuciones diferenciales por vía de las costumbres, suelen representar modelos valorativos diferentes, juegan papeles distintos en los mitos, ellas sufren una desventaja histórica respecto a ellos en lo concerniente a derechos civiles adquiridos.

Así, como concluíamos en Pacheco (2016), sorprende "la capacidad del patriarcado para ocultar su acción ejecutiva, su mandato, en el espacio intersubjetivo, siendo éste un plano de estudio inaccesible para las encuestas". Estas últimas, como toda herramienta de análisis positivista, sólo presentan la capacidad de explorar los niveles performativos del género, "dejando a los sujetos la posibilidad de enmascarar individualmente su sexismo bajo la asunción de roles, prácticas, narraciones e incluso actitudes perfectamente igualitarias" (Pacheco, 2016).

Esta denuncia, planteada inicialmente contra el diagnóstico cuantitativo del género, puede hacerse, en nuestra opinión, extensiva a todo tipo de iniciativas educativas de orientación objetivista implementables a gran escala, como el enfoque por competencias, pues, aunque puedan encontrarse soportadas por parámetros técnicos fiables, como la propuesta que realizábamos en Pacheco, Rodríguez-Díaz y García-Pérez (2013), carecen de la vocación y de la capacidad de acceder a la dimensión en la que se mantienen y perpetúan los significados machistas de que luego se sirven los sujetos para referenciar sus puestas en práctica. Atendiendo únicamente a estas últimas no sólo se obtendrá una visión distorsionada del problema, habida cuenta los impedimentos que encuentran los sujetos para cuestionar los significados que ponen en práctica, sino que dichos significados aparecerán simplificados por vía de la estandarización para su correcta integración como objeto de mediciones con vocación proyectiva, lo que favorecerá que la visión final tienda al optimismo, pues al fin y al cabo lo se que alcanza a medir es una interobjetividad en la que los referentes semánticos vienen dados autoritariamente por el sistema mismo.

En efecto, aquí defendemos que ese trasfondo encuentra por vocación la fijación de significados canónicos, inmunes a ser contestados por la práctica reflexiva de los sujetos de las representaciones, quiénes los toman como referente fundamental para la construcción de éstas. En educación, disponemos de valiosos trabajos 


\section{autêntica}

como el de Crawford y Chaffin (1997), quienes desvelan este fenómeno en el profesorado. Para estas autoras, el profesorado presenta la tendencia de atender diferenciadamente a chicos y chicas, aunque crean hacerlo de forma verdaderamente igualitaria, y sin manifestar conciencia alguna de que un buen número de tareas, intereses, comportamientos e incluso características físicas que son adscritas tradicionalmente a hombres y mujeres, en realidad se manifiestan de forma culturalmente contraria, siendo, por tanto, percibidas como inadecuadas.

En el caso del género, la conceptualización de este trasfondo arroja luz sobre cómo se instituye esa arquitectura cognoscitiva cargada de significados que son asumidos "por defecto" a modo de requerimiento técnico $y$, por ende, pretendidamente neutro, los cuales discriminan a las mujeres en el plano representacional. Un buen ejemplo de ello lo encontramos en el nivel lingüístico: reducido por la citada racionalidad cognoscitiva a la dimensión estructural-semántica de la puesta en práctica, en él se blanquean significados que excluyen a las mujeres pero que se presentan como neutros al amparo de un pretendido requerimiento técnico, impuesto sistémicamente desde una autoridad oficial u oficiosa, con poderes prescriptores o sin ellos. Este el caso del llamado masculino genérico, el cual puede llegar a ser objeto de debate en el conjunto de lenguas romance en plena confrontación con el inglés, esta última tradicionalmente considerada neutra respecto al género, aunque de forma equivocada, por la aplicación de ese mecanismo de razonamiento que, con anterioridad, exponíamos. El mal llamado masculino genérico es en realidad un masculino "por defecto", convirtiendo a la mujer en objeto de la justificación mientras no se demuestre lo contrario, o, si se prefiere, haciéndola objeto de implicatura conversacional (aunque Grice de ningún modo estuviera pensando en la igualdad de género).

Este mismo esquema se aplica cotidianamente a todo tipo de acciones socializadoras formales que son llevadas a cabo por el profesorado. Así, a pesar de que el procedimiento técnico representado por el marco competencial se presenta como garante de la neutralidad del contenido, lo cierto es que profesorado y alumnado encontrarían revolucionario ilustrar con mujeres las distintas etapas de la evolución humana o tomar como modelo anatómico de los músculos o del esqueleto a una mujer. Nuevamente, el procedimiento técnico garantiza el no cuestionamiento de la neutralidad, el masculino por defecto se presenta como masculino genérico para asegurar el despliegue de la puesta en práctica ya depurada de impedimentos "accesorios", sin mayores obstáculos que los condicionantes de orden técnico.

En definitiva, la arquitectura cognoscitiva a la que los agentes educativos, en especial el profesorado y, dentro de éste, aquél en formación inicial, acceden tras "pensar" y "hacer pensar" por competencias, fomenta la proliferación de iniciativas metodológicas incapaces de explorar los planos intersubjetivos de generación y compartición de significados sobre los que la desigualdad de género se asienta durante la puesta en práctica de actividades educativas. 


\section{autêntica}

DOI https://doi.org/10.31639/rbpfp.v12i24.356

\section{Consideraciones finales}

Nuestra reflexión ha tratado de poner en evidencia que el discurso de las competencias no promueve ni puede promover las herramientas conceptuales y analíticas adecuadas para la transformación de la sociedad hacia otra más igualitaria respecto al sexismo, a pesar de que puede presentarse como un procedimiento técnico, y, por ello, pretendidamente neutral, en relación a las ideologías de género. Hemos llevado a cabo este razonamiento respetando dos líneas argumentativas fundamentales, que son correlativas: 1) Desde el punto de vista del sujeto de una sociedad como la actual, las competencias promueven un tipo de conocimiento que depende de su puesta en práctica, esta última conducida por las lógicas productivas privilegiadas en el espacio público. Hemos descrito esto, siguiendo a Martínez-Bonafé (2004), como una colonización del sujeto, pues su capacidad reflexiva, característica desde la modernidad tardía (Giddens, 1991), quedaba mermada al no poder tomar el conocimiento al mismo tiempo como tema y como problema (Beck, 1998), quedando de este modo canalizada de acuerdo con lógicas productivas, ahora ya hegemónicas. La neutralización del aparato reflexivo afecta especialmente a la dimensión social del conocimiento y, por consiguiente, a las mujeres; y 2) el dicurso de las competencias como sistema cognoscitivo favorecedor de configuraciones normativas espaciales de corte estático y aspiraciones axiomáticas, reforzadoras de una canonicidad histórica (y desigual) y, por tanto, abocadas a su reproducción, que se afirman desde herramientas metodológicas y didácticas de tipo objetivista mantenedoras de significados prediscursivos y divulgadoras de un falso optimisto respecto a la desigualdad. Estos significados son tomados por el profesorado como referente para construir y difundir, de forma ya canalizada y sin capacidad para detectar los mandatos del patriarcado, las representaciones de género.

Nuestro trabajo concluye invitando al desarrollo de dos grandes líneas de razonamiento: una primera de tipo sociológico, con vocación de análisis sobre cómo el desmantelamiento de los estados del bienestar europeos coincide con un auge en la influencia en educación de los organismos internacionales, los cuales plantean sistemas de producción del conocimiento articulados en torno a rankings y clasificaciones mantenidos por agencias nacionales expertas, como las de acreditación, fomentando el predominio de publicaciones de corte descriptivo en la ciencia social, incapaces, en nuestra opinión, de aportar soluciones a problemas fundamentales como la desigualdad de género; y una segunda de tipo psicológico, que ponga el foco en los sujetos de la comunidad educativa, y en las formas alternativas de apropiación, internalización y exteriorización que ponen en marcha, no sólo en un sentido procedimental sino también actitudinal, frente a estos planteamientos absolutistas y estatizadores, de vocación estructurante del pensamiento social y reproductora de la desigualdad. 


\section{autêntica}

DOI https://doi.org/10.31639/rbpfp.v12i24.356

\section{REFERENCIAS BIBLIOGRÁFICAS}

BARKER, Roger G.Habitats, Environments and Human Behavior. San Francisco: Jossey-Bass, 1978.

BECK, Ulrich. La Sociedad del Riesgo: hacia una nueva modernidad. Barcelona: Paidós, 1998

BOURDIEU, Pierre. La Domination Masculine. Paris: Éditions du Seuil, 1998.

BRUNER, Jerome. Actos de Significado. Más allá de la revolución cognitiva. Madrid: Alianza Editorial, 1991.

BUTLER, Judith. Gender Trouble: Feminism and the subversion of identity. Nueva York: Routledge, 1990.

CASTELLS, Manuel. The Information Age: Economy, Society and Culture. Vol 2: The Power of Identity. Cambridge: Blackwell, 1997.

CRAWFORD, Mary, CHAFFIN, Roger y FITTON, Lori. Cognition in social context. Learning and Individual Differences, v. 7, n. 4, p. 341-362, 1995.

CRAWFORD, Mary y CHAFFIN, Roger. The meanings of difference: cognition in social and cultural context. En CAPLAN, P. CRAWFORD, M, J. HYDE, Shibley, RICHARDSON, J. (Eds.). Gender differences in human cognition. (p.81-130) Oxford: Oxford University Press, 1997.

DELAY, Béatrice. La transmission des savoirs dans l'entreprise: construire des espaces de coopération entre les générations au travail. Informations sociales, v. 134, n. 6, p. 66-77, 2006.

FOUREZ, Gérard. La construcción del conocimiento científico: Sociología y ética de la ciencia. Madrid: Narcea, 1994.

GIDDENS, Anthony. Modernity \& Self-Identity: Self \& Society in the Late Modern Age. Cambridge: Polity Press, 1991.

GRICE, Paul. Studies in the Way of Words. Cambridge: Harvard University Pres, 1989.

HABERMAS, Jürgen. Der philosophische Diskurs der Moderne: 12 Vorlesungen. Frankfurt: Suhrkamp Verlag, 1985.

LAMO DE ESPINOSA, Emilio. La sociedad reflexiva: Sujeto y objeto del conocimiento sociológico. Madrid: CIS, 1990.

LUTZ, Catherine A. y ABU-LUGHOD, Lila. Language and the politics of emotion. Cambridge: Cambridge University Press, 1990.

MARTíNEZ-BONAFÉ, Jaume. La Formación del Profesorado y el discurso de las competencias. Revista Interuniversitaria de Formación del Profesorado, v. 18, n. 3, p. 127-143, 2004. 


\section{autêntica}

DOI https://doi.org/10.31639/rbpfp.v12i24.356

OUELLET, Amélie. Le transfert des savoirs dans I'enseignement: une nécessité!Rimouski: Université du Québec, 2007.

PACHECO, Ramón, RODRÍGUEZ DÍAZ, Rosario y GARCÍA-PÉREZ, Rafael. Impacto de las brechas de género ygeneracional en la construcción de actitudes en padres y madres frente a las innovaciones coeducativas. Revistade currículum y formación del profesorado, 17, 181-200, 2013.

PACHECO, Ramón. Explorando la Brecha de Género en la Familia: Posicionamientos de Padres y Madres hacia la Coeducación y la Igualdad en la Escuela. (Tesis doctoral). Universidad de Sevilla - Sevilla, 2016.

PÉREZ-GÓMEZ, Ángel. Reinventar la escuela, cambiar la mirada. Cuadernos de Pedagogía, n. 368, p. 66-71, 2007.

PÉREZ-GÓMEZ, Ángel. ¿Competencias o pensamiento práctico? La construcción de los significados de representación y de acción. En GIMENO-SACRISTÁN, J (Comp.), Educar por competencias, ¿qué hay de nuevo? (p. 59-102). Madrid: Morata, 2008.

RUBIN, Gayle. The Traffic in Women: Notes on the Political Economy of Sex. En REITER, Rayna (Comp.), Toward and anthropology of women. Nueva York: Monthly Review Press, 1975.

WEBER, Max. La ética protestante y el espíritu del capitalismo. Madrid: Akal, 1998.

PACHECO, R. ; ZUBIAURRE, E. Igualdad de Género y Enfoque por Competencias: dificultades en la formación del profesorado. Formação Docente - Revista Brasileira de Pesquisa sobre Formação de Professores. Belo Horizonte. Vol. 12, nº 24 (p. 65-76) 31 ago. 2020 